\title{
The Basement Membrane Components Nidogen and Type XVIII Collagen Regulate Organization of Neuromuscular Junctions in Caenorhabditis elegans
}

\author{
Brian D. Ackley, ${ }^{1,2,3}$ Seong Hoon Kang, ${ }^{1}$ Jennifer R. Crew, ${ }^{1}$ Chris Suh, ${ }^{3}$ Yishi Jin, ${ }^{2,3}$ and James M. Kramer ${ }^{1}$ \\ ${ }^{1}$ Department of Cell and Molecular Biology, Northwestern University Medical School, Chicago, Illinois 60611, and ${ }^{2}$ Howard Hughes Medical Institute, and \\ ${ }^{3}$ Department of Molecular, Cellular, and Developmental Biology, University of California Santa Cruz, Santa Cruz, California 95064
}

\begin{abstract}
Vertebrate neuromuscular junctions (NMJs) contain specialized basal laminas enriched for proteins not found at high concentrations extrasynaptically. Alterations in NMJ basement membrane components can result in loss of NMJ structural integrity and lead to muscular dystrophies. We demonstrate here that the conserved Caenorhabditis elegans basement membrane-associated molecules nidogen/entactin (NID-1) and type XVIII collagen (CLE-1) are associated with axons and particularly enriched near synaptic contacts. NID-1 is concentrated laterally, between the nerve cord and muscles, whereas CLE-1 is concentrated dorsal to the ventral nerve cord and ventral to the dorsal nerve cord, above the regions where synapses form. Mutations in these molecules cause specific and distinct defects in the organization of neuromuscular junctions. The mutant animals exhibit mild movement defects and altered responses to an inhibitor of acetylcholinesterase and a cholinergic agonist, indicating altered synaptic function. Our results provide the first demonstration that basement membrane molecules are important for NMJ formation and/or maintenance in C. elegans and that collagen XVIII and nidogen can have important roles in synapse organization.
\end{abstract}

Key words: synaptogenesis; neuromuscular junction; extracellular matrix; collagen XVIII; nidogen; Caenorhabditis elegans

\section{Introduction}

Chemical synapses consist of a presynaptic terminal specialized for transmission of signals and a postsynaptic element with receptors for transducing signals. The well studied vertebrate neuromuscular junction (NMJ) (Sanes and Lichtman, 1999) is formed by a presynaptic neuron, a Schwann cell, and a postsynaptic muscle cell. These cells are separated by a specialized basement membrane (BM) within the NMJ synaptic cleft (Patton et al., 1998; Sanes and Yamagata, 1999). The major BM components are laminins (Colognato and Yurchenco, 2000), collagen types IV, XV, and XVIII (Kuhn, 1995; Myers et al., 1996; Musso et al., 1998), nidogens (also referred to as entactins) (Durkin et al., 1988; Mann et al., 1989; Kohfeldt et al., 1998), and heparan sulfate proteogylcans (HSPGs) (Yurchenco and Schittny, 1990; Timpl, 1994).

Laminins are heterotrimeric molecules consisting of noncovalently associated $\alpha, \beta$, and $\gamma$ chains. $\beta 2$ chain-containing laminins are highly concentrated within the synaptic cleft and are not found at high concentration extrasynaptically (Sanes and Lichtman, 1999). The nidogen G3 domain binds the laminin $\gamma 1$ chain with high affinity, whereas its G2 domain associates with type IV collagen and perlecan, which are also present within the synaptic cleft (Chiu and Ko, 1994; Sanes, 1997; Peng et al., 1999). Nidogen,

Received July 22, 2002; revised Jan. 30, 2003; accepted Feb. 4, 2003.

This work was supported by grants from the National Institutes of Health (NIH) (J.M.K., Y.J.). Y.J. is an Assistant Investigator of the Howard Hughes Medical Institute. We thank M. Nonet and A. Alfonso for providing antibodies. Some strains used in these studies were provided by the Caenorhabditis elegans Genetics Center, which is supported by the NIH Center for Research Resources.

Correspondence should be addressed to James M. Kramer, Department of Cell and Molecular Biology, Northwestern University Medical School, 303 East Chicago Avenue, Chicago, IL 60611. E-mail: jkramer@northwestern.edu. Copyright $\odot 2003$ Society for Neuroscience $\quad$ 2270-6474/03/233577-11\$15.00/0 collagen type IV, and perlecan are not restricted to the synaptic basal lamina but are broadly distributed in BMs.

The synaptic BM is essential for proper NMJ formation. Agrin, an HSPG, is required for acetylcholine receptor clustering, an early event in NMJ formation (Campanelli et al., 1992). Additional evidence for extracellular matrix (ECM) function in synapse formation comes from identification of muscular dystrophies caused by mutations in synaptic BM components (laminin B2) (Sunada et al., 1995), cell surface matrix receptors (integrin $\alpha 7$ ) (Burkin and Kaufman, 1999), and proteins linking these receptors to the cytoskeleton, e.g., dystrophin (Nonaka, 1998; Colognato and Yurchenco, 1999; Cohn and Campbell, 2000; Burkin et al., 2001). Loss of either laminin $\alpha 2$ or $\beta 2$ chain results in gross NMJ abnormalities, whereas loss of $\alpha 4$ results in misregistration of presynaptic and postsynaptic structures (Noakes et al., 1995; Allamand et al., 1997; Patton et al., 2001). BM proteins can clearly have specific roles in the organization and function of neuromuscular junctions.

Type XV and XVIII collagens are closely related, widely expressed BM molecules that have N-terminal thrombospondinlike procollagen domains, collagenous domains with multiple interruptions, and highly conserved C-terminal NC1 domains (Kivirikko et al., 1994; Rehn and Pihlajaniemi, 1994). Collagen XVIII is expressed in the developing nervous system and found on peripheral axons (Musso et al., 1998). Humans and mice with mutations in type XVIII collagen show several eye abnormalities (Sertie et al., 2000; Fukai et al., 2002). In Caenorhabditis elegans, collagen XVIII has been shown to affect cell motility and axon guidance via the NC1/endostatin domains (Ackley et al., 2001; Kuo et al., 2001). Mice lacking the closely related collagen XV have mild skeletal muscle myopathy and increased susceptibility 
to cardiac injury (Eklund et al., 2001; Fukai et al., 2002). Neither collagen XVIII nor collagen XV has been reported to accumulate specifically at vertebrate synapses, nor has any role been described for these molecules at the NMJ.

Vertebrate nidogens are encoded by two genes, nidogen- 1 and nidogen-2 (Kohfeldt et al., 1998). No overt phenotype was originally reported for mice deficient for nidogen-1 (Murshed et al., 2000) or nidogen-2 (Schymeinsky et al., 2002). However, a recent report indicates that nidogen-1-deficient mice display a loss of hindlimb motor control and spontaneous seizure-like symptoms, suggesting a deficit in nervous system function (Dong et al., 2002). Although both nidogens are widely distributed, a specific glycosylation form of nidogen-1 has been reported to be present at NMJs (Chiu and Ko, 1994).

Mutations in several C. elegans BM genes have been characterized, including type IV collagen chains emb-9 and let-2 (Guo et al., 1991; Sibley et al., 1994; Gupta et al., 1997), nidogen nid-1 (Kang and Kramer, 2000; Kim and Wadsworth, 2000), perlecan unc-52 (Rogalski et al., 1995), and type XVIII collagen cle-1 (Ackley et al., 2001). NID-1 and CLE-1 are associated with the nervous system (Kang and Kramer, 2000; Ackley et al., 2001), whereas EMB-9, LET-2, and UNC-52 are not (Sibley et al., 1994; Graham et al., 1997; Mullen et al., 1999). Deletion of the NC1 domain of cle-1 can cause defects in the migration of several neurons (Ackley et al., 2001). Mutations in nid-1 have been shown to cause defects in positioning of axons in the sublateral and ventral nerve cords (Kim and Wadsworth, 2000). unc-52, emb-9, and let-2 are required for viability and affect the development of several tissues, including the body wall muscles and pharynx (Mackenzie et al., 1978; Guo et al., 1991; Gupta et al., 1997). None of these BM proteins have previously been shown to have a role in synaptogenesis in C. elegans. We show here that CLE-1 and NID-1 are enriched near synapse-rich regions of the nervous system and are required for the proper organization of presynaptic zones in $C$. elegans. Mutations in cle-1 and nid-1 result in distinct defects in synapse organization and function, providing the first genetic evidence that $\mathrm{BM}$-associated proteins are important for C. elegans synaptogenesis.

\section{Materials and Methods}

Culture conditions. C. elegans culture and manipulation were performed using standard methods (Brenner, 1974). All strains were maintained at $20-23^{\circ} \mathrm{C}$, unless specifically stated otherwise. The following strains were used: wild-type N2 var. Bristol, CH118 nid-1(cg118), CH119 nid1(cg119) (Kang and Kramer, 2000), CH120 cle-1(cg120) (Ackley et al., 2001), CZ477 cle-1(ju34); juIs1, CB1072 unc-29(e1072) (Lewis et al., 1980a). The following integrated green fluorescent protein (GFP) neural marker strains were used: juIs1 [ $\left.\mathrm{P}_{\text {unc- } 25}:: \mathrm{SNB}-1:: \mathrm{GFP}\right]$ (Hallam and Jin, 1998); juIs76 [ $\left.\mathrm{P}_{\text {unc-25 }}: \mathrm{GFP}\right]$ (Huang et al., 2002).

PCR and sequencing. The cle-1(ju34) allele was sequenced as described (Ackley et al., 2001). The primers for detecting the ju34 deletion are as follows (nucleotide numbering from F39H11; GenBank AF164959): ex9F1 5'-GCCCCGCAGCTAGAGGTTTA-3' (21559-21578) and ex9R1 5'AACAATGCGAAGTGGCGATAC-3' (22821-22841). Sequencing of genomic DNA from ju34 animals identified a discontinuous deletion in exon 17 of cle-1, removing nucleotides (nts) 22112-22333 and 22341-22467 and leaving seven nts, 22334-22340, intact (numbering based on cosmid F39H11; GenBank AF164959).

Mosaic analysis. Mosaic analysis was performed as described (Zhen and Jin, 1999) using nuclear SUR-5::GFP as a marker for the array (Gu et al., 1998). Four animals that had lost the array in the muscle lineage were examined. Although no animals were generated that lost the array from the entire neural lineage, two animals were examined in which the array had been lost from large regions of the ventral cord.
Microscopy. Live epifluorescence microscopy was performed as described (Zhen and Jin, 1999; Ackley et al., 2001). Fluorescent and Nomarski images were obtained using a Zeiss Axiophot microscope equipped with a Photometrics Sensys CCD camera. Images were serially deconvolved using MicroTome software (VayTek, Fairfield, IA). Confocal images were acquired using a Zeiss LSM5 and processed using the Pascal Software (Zeiss).

Immunohistochemistry. Immunohistochemistry was performed as described (Finney and Ruvkun, 1990; Bettinger et al., 1996; Koushika et al., 2001). The antibodies used in this report are CeCol18 (Ackley et al., 2001), Ab1095 (SNT-1) (Nonet et al., 1993), $\alpha$ UNC-17 (Alfonso et al., 1993), and $\alpha$ NID-1 (Kang and Kramer, 2000).

Thrashing assay. Thrashing assays were based on Miller et al. (1996). Animals were picked into $50 \mu \mathrm{l}$ of M9 buffer in individual wells of a 96-well cell-culture dish, with the experimenter blind to the genotype. Animals were allowed to equilibrate for $2 \mathrm{~min}$ and then observed for 2 min. A thrash was counted as a change of direction in body movement.

SNB::GFP quantification. Images were quantified in Scion Image (Scion Corporation) after thresholding using the wand autocount function. Images were acquired on either a Zeiss LSM5 confocal microscope or a Zeiss Axioskop and saved as TIFF images with a scale bar. The images were opened in Scion Image and converted to a binary image using the thresholding command. A line was used to measure the number of pixels along the scale bar that was used to set the pixels per micrometer scale in the program. The wand autocount counts the number of white pixels surrounded by black pixels in the thresholded image to arrive at the area. These numbers were exported to Microsoft Excel for statistical analyses.

Axon guidance. The juIs76 GFP marker (Huang et al., 2002) illuminates the cell bodies and processes of the 6 dorsal type D (DD) and 13 ventral type $\mathrm{D}(\mathrm{VD})$ motor neurons. The arrangement of the cell bodies in the ventral nerve cord provides 10 distinct regions to score axon guidance. VD processes (axons) abut but do not overlap with other VD processes, only DD processes (dendrites) and vice versa. Thus each region can be simplified to correspond to an overlap of a single VD axon with a single DD dendrite. We examined 48 animals (480 regions) of each genotype for defasciculation defects in the ventral cord. Numbers are presented as percentage of regions that showed a defasciculation defect, indicating that a process was displaced from the edge of the muscle where it would normally make synapses.

Video microscopy. An $\sim 1 \times 1 \mathrm{~mm}$ square was drawn on a glass slide using a Pap pen (hydrophobic slide marker; Sigma, St. Louis, MO). M9 buffer $(10-15 \mu \mathrm{l})$ was used to fill the square. Animals were picked into the liquid and allowed to equilibrate for $2 \mathrm{~min}$. Images were acquired using the VidCap program (Freeware) using a Panasonic WV-CD110 analog camera on a Zeiss Axiovert 35M inverted microscope. Movies were edited to current length using Adobe Premiere 5.1.

Pharmacologic assays. Levamisole-induced egg-laying assays were performed as described (Kim et al., 2001). Aldicarb resistance assays were done as described (Jorgensen et al., 1995). Briefly, adult animals were scored for body movement or pharyngeal pumping after $8 \mathrm{hr}$ on normal growth medium containing the indicated concentration of aldicarb. Lethality was scored as a complete cessation of movement and pumping. Animals were scored as positive for pumping if they demonstrated continuous, vigorous pumping. Movement was scored as positive if any body wall muscle activity could be observed.

\section{Results}

\section{CLE-1 and NID-1 are enriched near synapses}

Most synapses in C. elegans form en passant along axons and are observed as bulging varicosities along the processes (White et al., 1986). The ventral and dorsal nerve cords are situated between a medial epidermal (hypodermal) ridge and the more laterally positioned body wall muscles (Fig. $1 A$ ). Body wall muscles extend processes, called muscle arms, to the nerve cords to receive innervation. In the ventral nerve cord, neuromuscular junctions are restricted to the lateral edge of the right fascicle, where it contacts the basement membrane at the muscle edge (Fig. $1 B$ ). The dorsal 

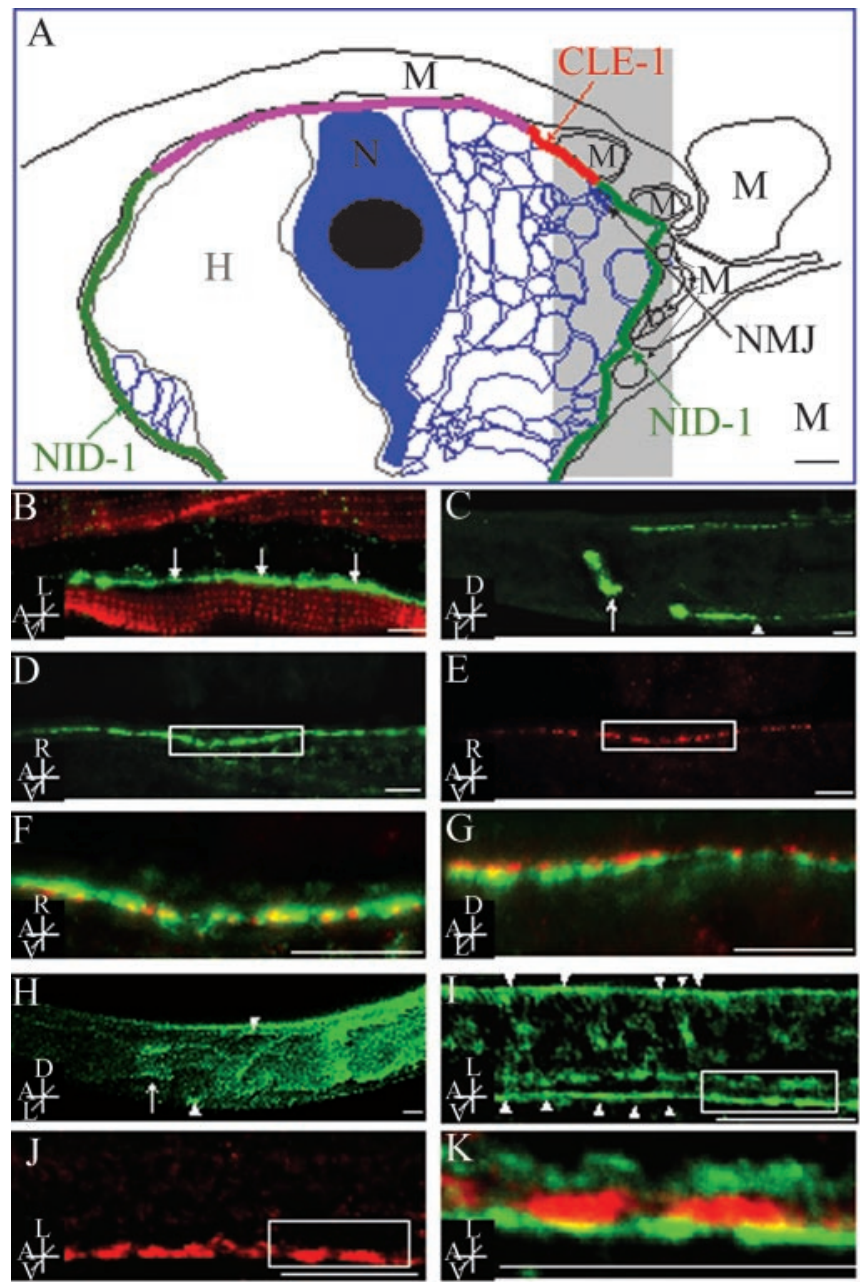

Figure 1. CLE-1 and NID-1 are concentrated near synaptic zones. $A$, Schematic representation of an electron micrograph cross section of the ventral nerve cord as viewed from a posterior perspective [based on White et al. (1986), their Figure 18]. From left to right are a muscle cell $(\mathrm{M})$, the left axon bundle (blue open circles), hypodermal ridge $(\mathrm{H})$, a neural cell body $(\mathrm{N})$, the right axon bundle (blue open circles), and another muscle cell (M). The left muscle is projecting a muscle arm $(M)$ over the nerve cord to the right fascicle. Other muscle arms in the section are observed as open black circles closely apposed to the right fascicle. The basal lamina covering the hypodermis and nerve cord is exaggerated in size. The general regions where CLE-1 (red) and NID-1 (green) are enriched are indicated. The remainder of the basal lamina (purple) can have lower levels of both CLE-1 and NID-1. This schematic represents projections of light microscopic data onto an EM scale and is for display purposes only. The gray shaded area indicates the approximate region where synapses are observed in $B$. A single neuromuscular junction (NMJ) present in the section is indicated, although NMJs can form anywhere along this side of the cord where neurons interface with muscle arms. A distinct basal lamina is present over the muscle cells but is not shown. Scale bar, $200 \mathrm{~nm}$. B, Synaptotagmin-1 (green) and perlecan (red) illustrate the position of synapses (arrows) relative to the muscles. Synapses are not evenly distributed in the region between the two muscle cells but rather are concentrated along the muscle edge. $(-K$, Immunolocalization of CLE-1 ( $C-G$, green) and NID-1 ( $H-K$, green) relative to the presynaptic protein UNC-17 (red) demonstrating the localization of the basement membrane proteins relative to cholinergic synapses. In all panels, anterior is to the left. Dorsal is up in $C, G$, and $H$, although the dorsal surface projects out in $D$ and $E$ and ventral projects out in $I-K$. Scale bars, $10 \mu \mathrm{m}$. C, Significant CLE-1 localization is observed along the axon process of the nerve ring (arrow) and the dorsal and ventral nerve cords (arrowheads). D, CLE-1 appears punctate along the nerve cords; the dorsal nerve cord is shown here. E, Anti-UNC-17 staining illustrates the presynaptic zone of cholinergic synapses along the dorsal cord. $F$, Merged image of boxed region from $D$ and $E$. CLE- 1 appears to be present along the surface of the cord and heavily concentrated over the synaptic region along the right muscle edge. The same pattern is seen on the ventral nerve cord (data not shown). G, A lateral perspective of the dorsal cord showing that CLE-1 (green) is localized ventral to the synaptic region defined by UNC-17 (red), on the pseudocoelomic face of the cord. H, NID-1 staining is observed on the nerve ring (arrow) and more weakly on the nerve cords (arrowheads) as well as on the pharynx ( $p$ ) and intestine (i). nerve cord is similarly organized, but on the left side of the midline.

The C. elegans nervous system is covered by a distinct basal lamina (White et al., 1986). However, the previously characterized basement membrane proteins, collagen IV and perlecan, have not been shown to be present in the neural basal lamina. We recently demonstrated that, in addition to being present in basement membranes throughout the animal, CLE-1 and NID-1 accumulate along the dorsal and ventral nerve cords and on the nerve ring (Kang and Kramer, 2000; Ackley et al., 2001). NID-1 is also associated with the sublateral nerve tracts. NID-1 and CLE-1 localize almost exclusively on nerve processes, not cell bodies, and associate with processes that travel anterior-posterior while generally being undetectable on dorsoventral processes, e.g., commissures, which do not contain synapses. NID-1 and CLE-1 staining is not necessarily distributed evenly along axon tracts but is strongest in the regions where synapses are abundant and is often in a punctate pattern reminiscent of synaptic structures (Kang and Kramer, 2000; Ackley et al., 2001).

To determine the localization of NID-1 and CLE-1 relative to synapses, we performed double-labeling experiments with antibodies against CLE-1 or NID-1 and a vesicular acetylcholine transporter, UNC-17, which is present in cholinergic presynaptic zones (Alfonso et al., 1993). At the light microscope level, it is not possible to determine whether NID-1 or CLE- 1 is present within or excluded from synaptic clefts. However, these molecules are present at higher concentrations along the lateral region of the nerve cord, where synaptic junctions are abundant, relative to the medial region, which has many fewer synapses. The CLE-1 and NID-1 proteins appear to be present on different faces of the nerve cords relative to synaptic domains (Fig. 1). NID-1 is present lateral to the region where synapses form, between the nerve cord and the muscle. CLE-1 is concentrated between the nerve cords and the pseudocoelomic space or overlying muscle arms, i.e., along the dorsal face of the ventral nerve cord and the ventral face of the dorsal nerve cord (Fig. 1).

Although CLE-1 and NID-1 are found overlapping or closely apposed to UNC-17 along the nerve cords, we do not observe a consistent association of NID-1 or CLE-1 with UNC-17 puncta, and vice versa. UNC-17 is not present at all of the presynaptic zones in the nerve cords. We observe similar distributions of CLE- 1 and NID-1 relative to a marker for GABAergic presynaptic zones, juIs1 (see below). NID-1 and CLE-1 are also detected in nonsynaptic regions of the nerve cords, but generally at significantly lower levels. We conclude that NID-1 and CLE-1 are enriched in regions where synapses form but are not synapse specific.

\section{Mutations in cle-1 and nid-1 cause defects in synaptotagmin localization}

Synaptotagmin is an endogenous component of synaptic vesicles found in all presynaptic zones (Nonet et al., 1993). To examine the significance of the CLE-1 and NID-1 associations with nerve cords, we examined the localization of synaptotagmin (SNT-1) in cle-1 and nid-1 mutant animals. We examined the cle-1(cg120)

I, NID-1 localizes between the ventral muscle edges (arrowheads) and nerve cords. A similar pattern is observed on the dorsal cord (data not shown). The more medial staining seen in the top of the boxed region is NID-1 associated with the extracellular mantle of the mechanosensory neuron AVM. J, UNC-17 staining along the right fascicle of the ventral nerve cord. $K$, Merged image of the boxed regions from / and J. NID-1 is concentrated along the lateral edge of the synaptic region. Staining of the AVM mantle is again seen above the UNC-17 (red) staining. 


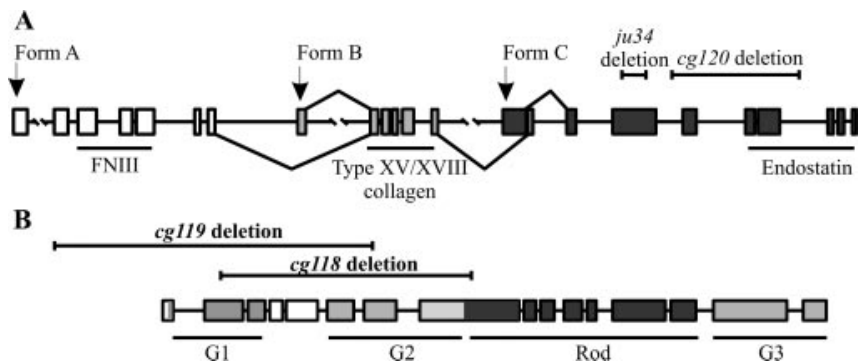

Figure 2. cle-1 and nid-1 gene structures and mutations. $A$, The cle-1 gene structure is shown with the different domains indicated in the three isoforms as reported previously (GenBank AF164959) (Ackley et al., 2001). CLE-1A-specific exons are white, those in forms $A$ and $B$ are light gray, and those common to all three isoforms are dark gray, with the exceptions that exon 8 is unique to CLE-1B and exon 14 is unique to CLE-1C. The ju34 deletion removes part of exon 17, which encodes the Gly-X-Y collagenous domain, and causes a premature stop codon. The $c g 120$ deletion removes exons $18-20$ and also causes a premature termination. $B$, The nid-1 gene structure is shown with the globular and rod domains indicated. The structure of nid-1 and the c 119 and c 1118 deletions have been reported previously (Kang and Kramer, 2000). The c 119 deletion removes exons 1-7 of the nid-1 coding region plus $948 \mathrm{bp}$ upstream of the ATG and is a molecular null for NID-1. The c 118 deletion is in frame and removes exons $2-8$, resulting in an NID-1 protein missing some of the $G 1$ and all of the $G 2$ domains, leaving the rod and $\mathrm{G} 3$ domain intact.

allele, which is a deletion of the NC1 domain (Ackley et al., 2001), and two alleles of nid-1, cg118, an in-frame deletion of the G2 domain, and $c g 119$, a deletion that removes the promoter and first six exons and is a molecular null (Kang and Kramer, 2000) (Fig. 2). In wild-type dorsal and ventral nerve cords, SNT-1 is observed as discrete lines along the muscle edges. These lines represent numerous puncta that appear to run together. Along the sublateral nerve cords SNT-1 appears as individual puncta (Fig. 3).

In the nerve cords of cle-1(cg120) mutant animals, the SNT-1 staining pattern was observed as clusters of larger puncta that are not as tightly associated with the muscle edges. Along the sublateral nerve cords the size of the puncta appeared enlarged in cle1(cg120) relative to wild-type animals. In nid-1(cg119) null animals, SNT-1 appears diffused along the longitudinal axis of the nerve cord, and individual puncta are not generally distinguishable in the dorsal or ventral nerve cords. nid-1(cg118) animals displayed enlarged synapses along the ventral and dorsal nerve cords that were also often diffused along the axis of the cords. Antibody staining for UNC-10, a presynaptic active zone component (Koushika et al., 2001), showed defects similar to those observed for the entire presynaptic zones (data not shown).

\section{A new cle-1 allele identified in a synapse-defective screen}

Genetic screens have isolated mutations that cause morphological defects in presynaptic zone formation by visualizing the synaptic vesicle protein synaptobrevin (SNB) fused to GFP (Nonet, 1999; Zhen and Jin, 1999; Schaefer et al., 2000; Zhen et al., 2000; Crump et al., 2001). The presynaptic zone of the 19 GABAergic ventral cord motor neurons can be visualized using the unc-25 promoter to drive expression of SNB::GFP (Jin et al., 1999; Nonet, 1999). This marker is observed as regularly sized and spaced individual puncta along the dorsal and ventral nerve cords (Fig. 4). These puncta represent the presynaptic zones where the DD and VD motor neurons innervate the dorsal and ventral muscles, respectively.

Using this marker, we identified a mutation, ju34, which results in larger fluorescent puncta in the GABAergic motor neurons. We mapped ju34 to the region of LG1 near cle-1 and found that it failed to complement the cle-1(cg120) allele. Additionally, a transgene carrying the cosmid $\mathrm{F} 39 \mathrm{H} 11$, which contains the complete wild-type cle-1 gene (Ackley et al., 2001), fully rescued the ju34 mutant phenotype. The ju34 mutation results in loss of 29 of the 40 Gly-X-Y repeats in CLE- 1 and places the downstream exons, which encode the NC1/endostatin domain, out of frame (for details, see Materials and Methods) (Fig. 2). Staining with an antibody directed against a CLE-1 epitope located C-terminal to the deletion, CeCol18 (Ackley et al., 2001), shows greatly reduced but detectable reactivity (data not shown). Similar results were obtained for the cle-1(cg120) deletion, which removes $45 \%$ of the epitope (Ackley et al., 2001). The small amount of CLE-1-specific immunoreactivity indicates that some protein can be produced and secreted, possibly via alternative splicing or read-through of the message, resulting in a reduced level of detectable protein. The two cle-1 alleles, cg120 and ju34, are strong loss of function, but do not appear to be null mutations.

\section{GABAergic NMJ defects in cle-1 mutants}

We further examined synaptic defects in cle-1 mutants using the Punc-25::SNB::GFP ( $j u I s 1)$ marker to visualize individual presynaptic zones of GABAergic motor neuron synapses. Both ju34 and $c g 120$ mutants showed similar abnormal SNB::GFP puncta that appeared enlarged and more widely spaced than normal, and a decrease in the number of puncta in both the dorsal and ventral cords (Fig. 4, Table 1). Wild-type animals display $\sim 150$ puncta in both the dorsal and ventral cords. cle-1(cg120) mutants display an average of 103 puncta in the ventral cord and 96 puncta in the dorsal cord, and ju34 animals display an average of 102 ventral and 97 dorsal puncta. We calculated the area of the presynaptic zones in wild-type and mutant animals using the NIH Image program (see Materials and Methods). In wild-type animals the areas of presynaptic zones are $0.83 \pm 0.96 \mu \mathrm{m}^{2}$ along the ventral cord and $0.85 \pm 0.36 \mu \mathrm{m}^{2}$ along the dorsal cord. By contrast, in cle-1(cg120) mutants the ventral cord puncta are $2.77 \pm 2.02$ $\mu \mathrm{m}^{2}$, and the dorsal cord puncta are $2.02 \pm 1.21 \mu \mathrm{m}^{2}$. In ju34 animals the puncta are even larger, with the ventral cord puncta $4.25 \pm 3.74 \mu \mathrm{m}^{2}$ and the dorsal cord puncta $3.31 \pm 1.21 \mu \mathrm{m}^{2}$. The puncta appear both longer and wider in $c g 120$ and ju34 animals (Fig. 5, Table 1). The ju34 and $c g 120$ alleles act like recessive, loss-of-function mutations, and heterozygous animals appear normal. The synaptic defects appear similar in each strain, although ju34 causes slightly larger puncta, suggesting that the defects may occur because of the loss of the NC1 domain, which is common to both mutations.

Because we observed both a reduction in number and an increase in size of the remaining SNB::GFP puncta, we asked whether this effect could result from simple fusion of existing puncta or represents an independent effect on synaptic organization. We examined the total measurable synaptic area over a region of the nerve cord, a value that should not be altered if fusion is the only alteration in the mutants. Over a $100 \mu \mathrm{m}$ distance, wild-type animals were observed to have $17.6 \mu \mathrm{m}^{2}$ of SNB::GFP fluorescence. cle-1(cg120) animals were observed to have 23.1 $\mu \mathrm{m}^{2}$ and cle-1(ju34) animals to have $46.3 \mu \mathrm{m}^{2}$ of fluorescence. These results suggest that the increase in synaptic area does not simply result from fusion of neighboring synapses, but rather involves a defect in synaptic morphology.

\section{GABAergic NMJ defects in nid-1 mutants}

Because NID-1 is also concentrated along the synaptic zone of the nerve cords and nid-1 mutations cause defects in synaptotagmin accumulation, we examined the two nid-1 mutations using 

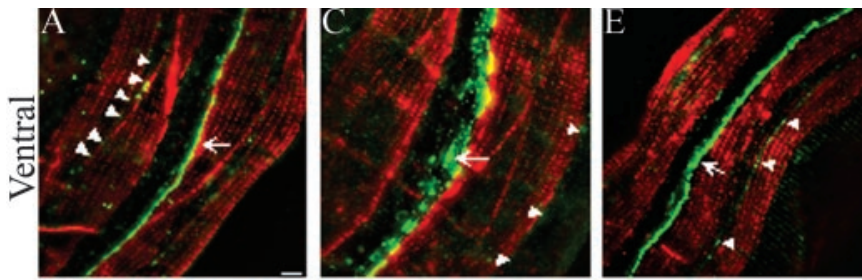

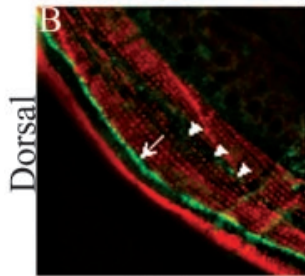

wild type

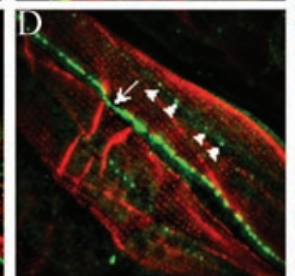

cle-1(cg120)

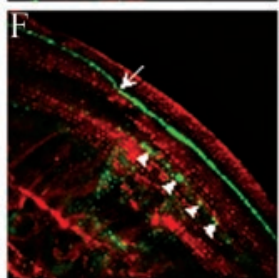

nid-1(cg119)
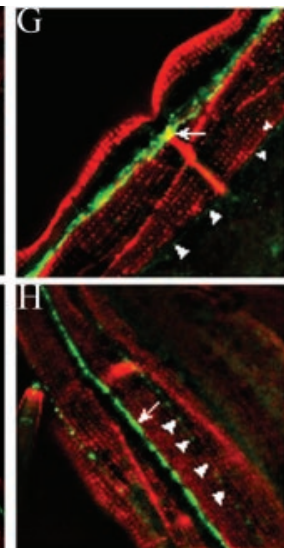

nid-1(cg118)
Figure 3. Synaptotagmin staining in cle-1 and nid-1 animals. The presynaptic zones of all synapses are illuminated by staining with anti-SNT-1 (green). In all panels anterior is down and ventral $(A, C, E, G)$ or dorsal $(B, D, F, H)$ projects out. Scale bar: (in $A) A-H$, $10 \mu \mathrm{m}$. Samples are costained with anti-UNC-52 perlecan (red) to highlight muscle cells. Arrows indicate the nerve cords, whereas arrowheads indicate the positions of the sublateral nerves. $A, B$, In wild-type animals, synapses in the ventral and dorsal nerve cords (arrows) appear as continuous, condensed lines of staining. Weaker staining is also observed in the presynaptic zones along the sublateral nerve cords (arrowheads). C, D, In cle-1(cg120) animals the SNT-1 staining appears more dispersed, and the puncta are enlarged relative to wild type. This dispersion of synapses is not a result of ventral cord defasciculation, which is rare in these animals. E, F, In nid-1(cg119) animals the SNT-1 staining appears more diffused, and in the sublateral nerve cord (arrowheads) puncta appear more frequently than in wild-type animals. G, H, In nid-1(cg118) animals SNT-1 staining appears more fragmented, and the puncta are somewhat dispersed.
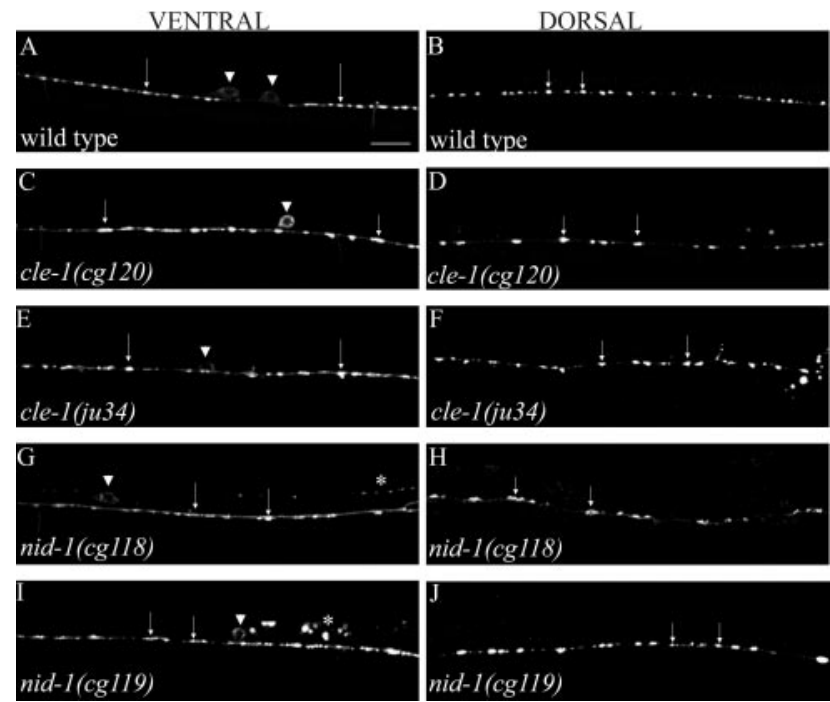

Figure 4. Mutations in cle-1 and nid-1 cause defects in presynaptic zones. Confocal images of presynaptic zones visualized with SNB::GFP (see Materials and Methods). In all panels anterior is left and ventral $(A, C, E, G, I)$ or dorsal $(B, D, F, H, J)$ is projecting out. Arrowheads indicate the position of cell bodies along the ventral cords; arrows indicate representative presynaptic puncta present in each panel. Scale bar: (in $A) A-J, \sim 10 \mu \mathrm{m}$. $A, B$, The ventral and dorsal cord presynaptic zones of wild-type animals. The GFP puncta (arrows) are of approximately equal size and spacing. $C, D$, cle-1(cg120) animals display enlarged puncta that are spaced farther apart than in wild type. $E, F$, Defects similar to those in c 120 are observed in cle-1(ju34) animals. $G, H$, nid-1(cg118) animals display enlarged puncta that are often more rounded than in cg119 animals. The puncta are also spread farther apart than in wild type. I, J, nid-1(cg119) animals display ventral puncta that often appear to run together or appear smaller and clustered closer together than in wild type. On the dorsal cord the puncta are more irregularly shaped but still often distended along the axis of the cord.

SNB::GFP and found both to be defective. In $\operatorname{cg} 119$ animals the puncta on the dorsal and ventral nerve cords are affected differently. Along the ventral nerve cord the puncta appear smaller and often smeared, with an apparent increase in the number of puncta. The average area of ventral puncta is $5.56 \pm 9.24 \mu \mathrm{m}^{2}$. The dorsal puncta appear more normal but are slightly enlarged, with an average area of $1.15 \pm 0.65$ $\mu \mathrm{m}^{2}$ (Figs. 4, 5, Table 1). The puncta in cg119 animals are a mix of small puncta and elongated puncta that appear three to four times longer than in wild-type. These puncta are likely to result from several smaller puncta that have run together, thus dramatically increasing the variability in size of puncta. The dorsal nerve cord exhibits similar small, smeary puncta interspersed with larger, clumped puncta, with no change in the number of puncta (Fig. 4, Table 1).

The NID-1 G2 domain deletion, $\operatorname{cg} 118$, has a different effect, with the puncta appearing more spread out and disorganized, occasionally enlarged, and often a decreased number of apparent puncta (Fig. 4, Table 1). Similar defects are observed on both the dorsal and ventral nerve cords. The average area of ventral puncta is $2.01 \pm 3.74 \mu \mathrm{m}^{2}$, and the area of dorsal puncta is $1.38 \pm 1.14 \mu \mathrm{m}^{2}$ (Fig. 5, Table 1).

Presynaptic defects are not necessarily secondary to axon positioning defects

Because axon guidance defects have been demonstrated in cle-1 and nid-1 mutants, we were curious about whether the synaptic defects were a secondary effect of positioning defects. We examined the penetrance of axon positioning defects along the ventral nerve cord in these animals using a GFP marker that reveals the cell bodies and processes of $6 \mathrm{DD}$ and $13 \mathrm{VD}$ motor neurons,

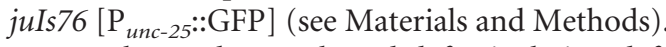

We observed ventral cord defasciculation defects in $23 \%$ of cle-1 animals. On average, in each animal that exhibited any defect only $2.7 \%$ of all axons were detectably defective. Fifty-two percent of nid-1 (cg118) and 50\% of nid-1(cg119) animals exhibited positioning defects, with an average of 6.6 and $5.4 \%$, respectively, of the axons examined exhibiting a defect. In contrast, synaptic defects were observed in $100 \%$ of the mutant animals and were seen throughout the nerve cords, not confined to single axons. These results suggest that defects in synaptic organization are present on axons that are not obviously mispositioned. Furthermore, the nature of the synaptic defects is distinct in $c l e-1$ and nid-1 mutants, indicating that they have some specific effect on synapse organization. These results make it very unlikely that the highly penetrant synaptic defects are simply the result of axon guidance defects.

\section{Similar presynaptic and postsynaptic defects in $c l e-1$ and nid-1 mutants}

An essential feature of synapses is the precise registry of the presynaptic and postsynaptic structures. Recent studies have demonstrated a role for the ECM in the coordinate formation of presynaptic and postsynaptic structures (Nguyen et al., 2000). We asked whether the presynaptic defects in cle-1 and nid-1 mutants were correlated with defects in the postsynaptic structures in muscle cells. We visualized GABAergic postsynaptic NMJ structures using a functional GFP-tagged GABA receptor, UNC- 
Table 1. SNB::GFP puncta number and size by genotype

\begin{tabular}{|c|c|c|c|c|c|}
\hline Genotype & Puncta & Area $\left(\mu \mathrm{m}^{2}\right)$ & $\begin{array}{l}\text { Perimeter } \\
(\mu \mathrm{m})\end{array}$ & Length $(\mu \mathrm{m})$ & Width $(\mu \mathrm{m})$ \\
\hline Wild type & $n=14$ & $n=9$ & & & \\
\hline Dorsal cord & $149 \pm 11$ & $0.85 \pm 0.36$ & $3.28 \pm 1.21$ & $1.30 \pm 0.41$ & $0.79 \pm 0.19$ \\
\hline Ventral cord & $153 \pm 12$ & $0.83 \pm 0.96$ & $3.92 \pm 2.67$ & $1.62 \pm 1.04$ & $0.57 \pm 0.25$ \\
\hline cle-1(cg120) & $n=20$ & $n=5$ & & & \\
\hline Dorsal cord & $96 \pm 8$ & $2.02 \pm 1.21$ & $6.58 \pm 3.11$ & $2.46 \pm 1.16$ & $1.01 \pm 0.32$ \\
\hline Ventral cord & $103 \pm 14$ & $2.77 \pm 2.02$ & $9.10 \pm 5.36$ & $3.52 \pm 2.18$ & $0.93 \pm 0.32$ \\
\hline cle-1(ju34) & $n=12$ & $n=4$ & & & \\
\hline Dorsal cord & $97 \pm 7$ & $3.31 \pm 1.21$ & $8.94 \pm 3.34$ & $3.69 \pm 1.64$ & $1.20 \pm 0.32$ \\
\hline Ventral cord & $102 \pm 11$ & $4.25 \pm 3.74$ & $9.44 \pm 5.17$ & $3.61 \pm 1.99$ & $1.33 \pm 0.721$ \\
\hline nid-1(cg119) & $n=13$ & $n=7$ & & & \\
\hline Dorsal cord & $154 \pm 12$ & $1.15 \pm 0.65$ & $4.44 \pm 1.86$ & $1.75 \pm 0.74$ & $0.76 \pm 0.21$ \\
\hline Ventral cord & $188 \pm 21$ & $5.56 \pm 9.24$ & $12.08 \pm 14.01$ & $4.18 \pm 4.64$ & $1.18 \pm 1.02$ \\
\hline nid-1(cg118) & $n=13$ & $n=5$ & & & \\
\hline Dorsal cord & $116 \pm 12$ & $1.38 \pm 1.14$ & $4.78 \pm 2.20$ & $1.81 \pm 0.85$ & $0.88 \pm 0.34$ \\
\hline Ventral cord & $143 \pm 14$ & $2.01 \pm 3.74$ & $5.73 \pm 5.32$ & $2.23 \pm 1.74$ & $0.83 \pm 0.45$ \\
\hline
\end{tabular}

49B (Bamber et al., 1999). We observed defects in the pattern of the UNC-49B::GFP marker that correlated with those seen with the SNB::GFP marker in cle-1 and nid-1 mutants (Fig. 6). In both cle-1 mutants the puncta appear enlarged, rounded, and spaced farther apart than normal. nid-1(cg119) animals exhibited elongated puncta interspersed with smaller smeary puncta. nid1(cg118) animals have a mix of small puncta and elongated puncta that are spread farther apart. These results demonstrate that both presynaptic and postsynaptic structures are affected in cle-1 and nid-1 mutants and reinforce the concept that synaptogenesis is a coordinated process between neurons and their target cells.

\section{Synaptic defects in cle-1 appear to function cell nonautonomously}

Because CLE-1 and NID-1 are secreted and incorporated into the extracellular matrix, we asked whether their expression was specifically required in neurons or muscles. We conducted mosaic analysis in cle-1(cg120) animals that carried a cle-1 $(+)$ cosmid and SUR-5::GFP as a marker for cells that either retained (GFP + ) or lost (GFP-) the extrachromosomal array (see Materials and Methods). Four animals that had lost the array from the muscle lineage were scored for SNB::GFP puncta shape and distribution and were found to be wild type. Two animals that had lost the array along large portions of the ventral nerve cord were also found to have wild-type puncta. Thus, the synaptic defects in cle-1(cg120) animals were rescued equally well when the array was lost from the muscle lineage or neural lineage, indicating that CLE-1 functions cell nonautonomously. Previous data have indicated that NID-1 also acts cell nonautonomously with regard to axon guidance (Kim and Wadsworth, 2000), because either muscle or neural-specific expression was capable of rescuing axonal defects. These results are consistent with previous reports for extracellular matrix molecules acting cell nonautonomously in $C$. elegans (Graham et al., 1997).

cle-1 and nid-1 exhibit movement defects in a thrashing assay The movement of cle-1 and nid-1 mutant animals on normal growth media plates appears essentially wild type, despite the highly penetrant synaptic structure defects described here and the axonal defects described previously (Kim and Wadsworth, 2000; Ackley et al., 2001). To assess whether these mutations could result in more subtle effects on movement, animals were examined using a thrashing assay (Miller et al., 1996). Thrashing is a

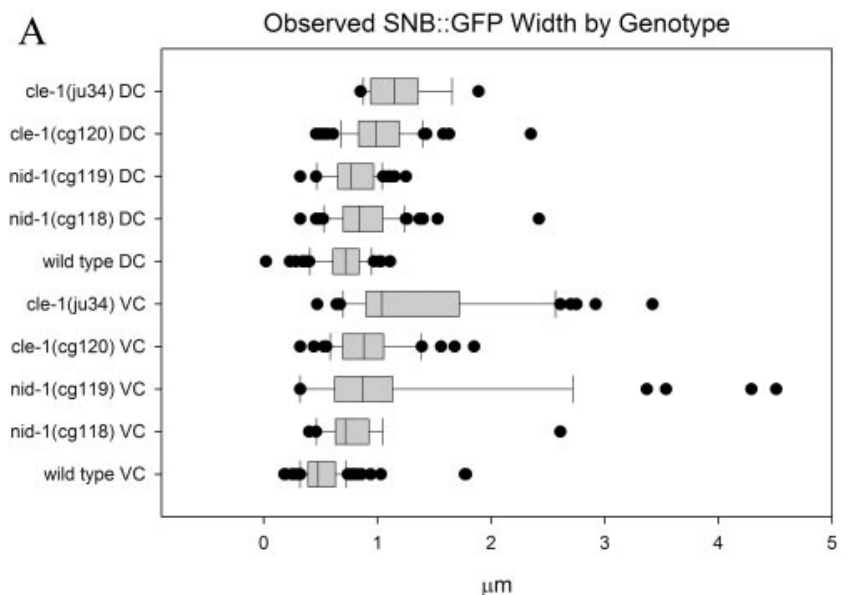

B

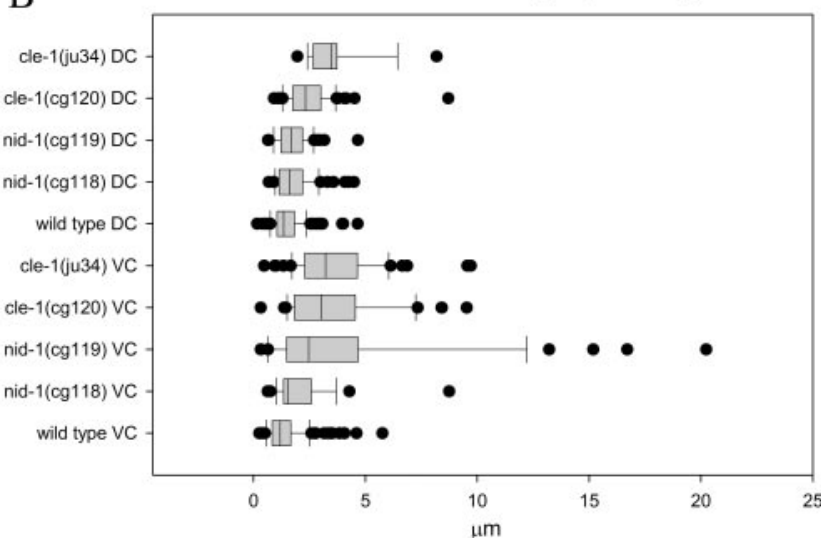

Figure 5. Presynaptic zones are longer and wider in cle-1 and nid-1 mutants. The individual measurements of the SNB::GFP puncta within the presynaptic zones for width $(A)$ and length $(B)$ are presented as box plots. The box represents those values that lie within $25-75 \%$ of the total population of individual synapse measurements, and within the box a line denotes the median value. The whiskers designate the $10-90 \%$ range with outliers shown as black dots. In each graph the ventral cord is represented in the top half of the graph, and the dorsal cord is represented in the bottom half. It is notable that the distribution of synapses seen in cle-1 mutant animals is shifted such that $>75 \%$ of all synapses are longer and wider than the median values observed for wild type. Furthermore, the median values for cle- 1 animals lie in the outlier regions for wild-type synapses. In nid-1/(cg119) null animals the ventral nerve cord is more greatly affected than the dorsal cord, leading to a dramatic variation in the length and width observed, although the length is most strongly affected. DC, Dorsal cord; VC, ventral cord. 

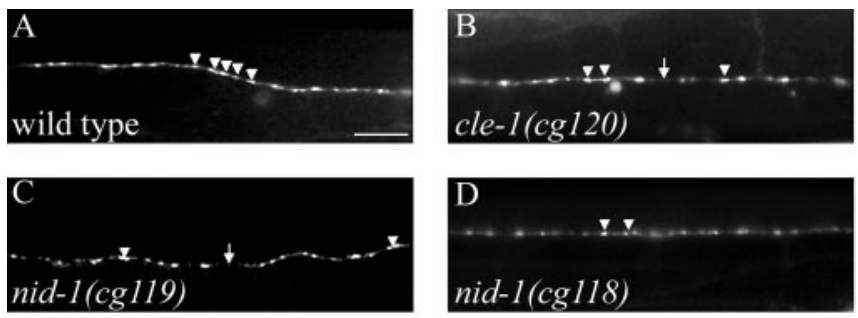

Figure 6. Postsynaptic defects in cle-1 and nid-1 mutants. A postsynaptic marker UNC-49B::GFP (Bamber et al., 1999), demonstrates that similar defects are observed in postsynaptic structures as shown for presynaptic structures. In all panels anterior is left and ventral is projecting out. Scale bar: (in $A) A-D, 10 \mu \mathrm{m}$. $A$, Wild-type animals display evenly sized, evenly spaced GFP puncta (arrowheads). B, cle-1 animals display larger puncta (arrowheads) that are separated by large gaps (arrow). C, nid-1(cg119) animals often display puncta that appear fused (arrowheads) and have gaps (arrow) along the cord. D, nid-1(cg118) animals have larger and more diffuse-appearing puncta (arrowheads).

high-frequency locomotory behavior that occurs when animals are placed in liquid. In this behavior there is a coordinated movement in which the animal brings the head and tail toward each other, flexing around the approximate midpoint with a regular amplitude and alternation between ventral and dorsal flexure (supplemental materials). Animals with defects in synaptic transmission exhibit reductions in the rate of thrashing behavior (Miller et al., 1996).

Wild-type animals thrash at an average rate of 162.5 thrashes per minute (TPM). A decrease in the rate of thrashing was observed for cle-1(cg120) (123 TPM) and nid-1(cg119) (130 TPM). Additionally, the mutant animals exhibited uncoordinated movements of the head and tail that were not observed in wildtype animals. For example, in cle-1(cg120) mutants the head and tail often move in opposite directions or one is static while the other is moving, behaviors that are very rarely observed in wildtype animals (Fig. 7) (supplemental movies; available at www. jneurosci.org). The mutants also frequently over-bend, such that the head and tail cross over one another. Interestingly, nid1 (cg118) $\Delta \mathrm{G} 2$ animals displayed an increase in thrashes per minutes (172 TPM) relative to wild-type, but with a decrease in the amplitude of the behavior (Fig. 7) (supplemental movies, available at www.jneurosci.org). cg118 animals also show uncoordinated movements of the head and tail, although at lower penetrance. Our results suggest that the axonal positioning and/or synaptic structure defects in cle-1 and nid-1 mutants can result in loss of normal coordination of body wall muscle contraction.

Because CLE-1 and NID-1 also accumulate under body wall muscles (Kang and Kramer, 2000; Ackley et al., 2001), it is possible that defects in muscle cell function contribute to the observed movement defects. However, staining for UNC-52 perlecan (Fig. 3 ) demonstrates that dense bodies are formed normally in the mutant animals. Furthermore, staining with myosin-specific antisera demonstrated no observable defects in the organization of thick filaments in mutant animals (data not shown). Given that we observe multiple defects in the organization of the nervous system, the most penetrant of which are synaptic, it is likely that the synaptic defects are a major cause of the movement defects detected in the thrashing assays.

\section{Response to a cholinergic agonist is altered in nid-1 and cle-1 mutants}

To assess whether synaptic transmission is altered in cle-1 and nid-1 mutants, we measured egg laying in response to the acetylcholine agonist levamisole (Lewis et al., 1980b, 1987; Waggoner
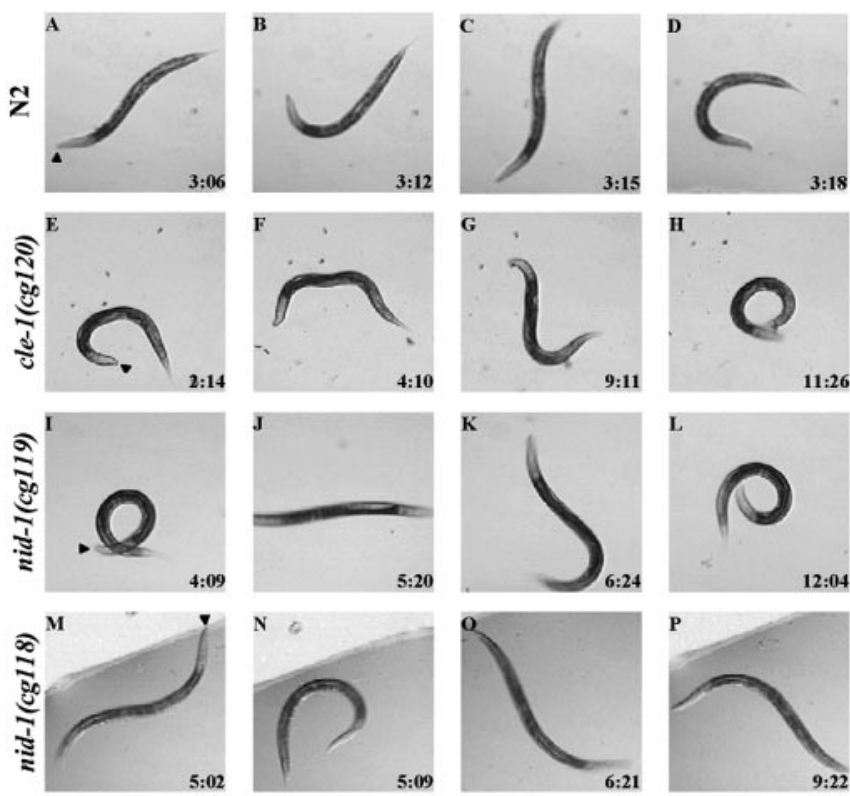

Figure 7. Thrashing movie stills. Still images taken from the thrashing movies (supplemental materials, available at www.jneurosci.org) of animals placed in M9 buffer are presented. The head of the animal is indicated with an arrowhead in the first panel for each genotype. The time from the movie is indicated in seconds at the bottom right of each panel. $A-D$, Wild-type animals flex around the mid-body region, bringing the head and tail toward each other and forming a "C-like" shape. E-H, cle-1(cg120) animals have a lower rate of thrashing and display uncoordinated movements of the head and tail $(G)$, as well as over-bending such that the head and tail cross $(H) . I-L$, nid-1(cg119) animals display over-bending $(I, L)$ and some uncoordinated movements of the head and tail $(K) . M-P$, nid-1(cg118) mutants often display abnormally shallow bending movements during which the amplitude of the movement is reduced. Occasional over-bending is also observed $(N)$.

et al., 2000). In the presence of levamisole, wild-type animals are stimulated to lay eggs in a dose-dependent manner. Both nid-1 mutants showed a strongly decreased egg-laying response, indicating reduced cholinergic transmission in the egg-laying muscles of these animals (Fig. 8 A, Table 2 ). cle- 1 animals have a highly variable response to levamisole. The mean number of eggs laid does not differ from wild type, but the range of responses is much greater than wild type (Table 2). At all but the lowest levamisole concentration, there are cle-1 mutants that lay more and fewer eggs than wild-type animals. cle-1 mutants are often egg-laying defective $(\mathrm{Egl})$, retaining larger than normal numbers of eggs in the uterus (Ackley et al., 2001), and stimulation of Egl animals could result in release of a larger number of eggs than normal. Notably, some cle-1 mutants fail to lay any eggs even at high levamisole concentrations, indicating that some animals are resistant. The increased range of egg laying, even at lower doses, indicates that $c l e-1$ animals respond differently to levamisole than wild-type animals, suggesting that cholinergic transmission is altered. We observe different responses to levamisole in cle-1 and nid-1 animals, indicating that the loss of each molecule causes distinct defects in cholinergic transmission.

\section{nid-1 and cle-1 mutants are resistant to aldicarb}

To further assess synaptic functional defects, we analyzed the response of mutant animals to the cholinesterase inhibitor aldicarb. When cultured on aldicarb-containing media for $8 \mathrm{hr}$, wildtype animals display a dose-dependent loss of movement, cessation of pharyngeal pumping, and lethality caused by accumulation of toxic levels of acetylcholine in synaptic clefts. Mutations that reduce cholinergic transmission show resistance 
A

\section{Egg Laying in Response to Levamisole}

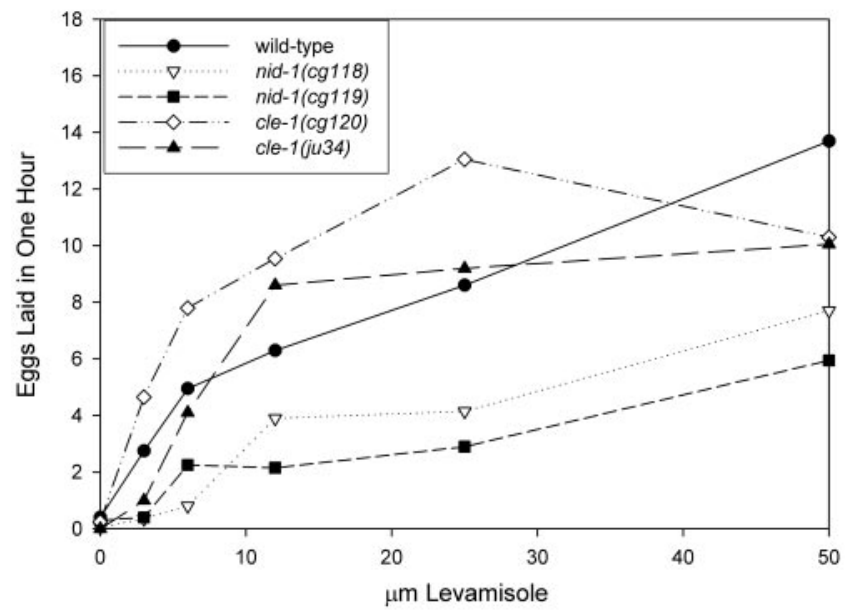

B

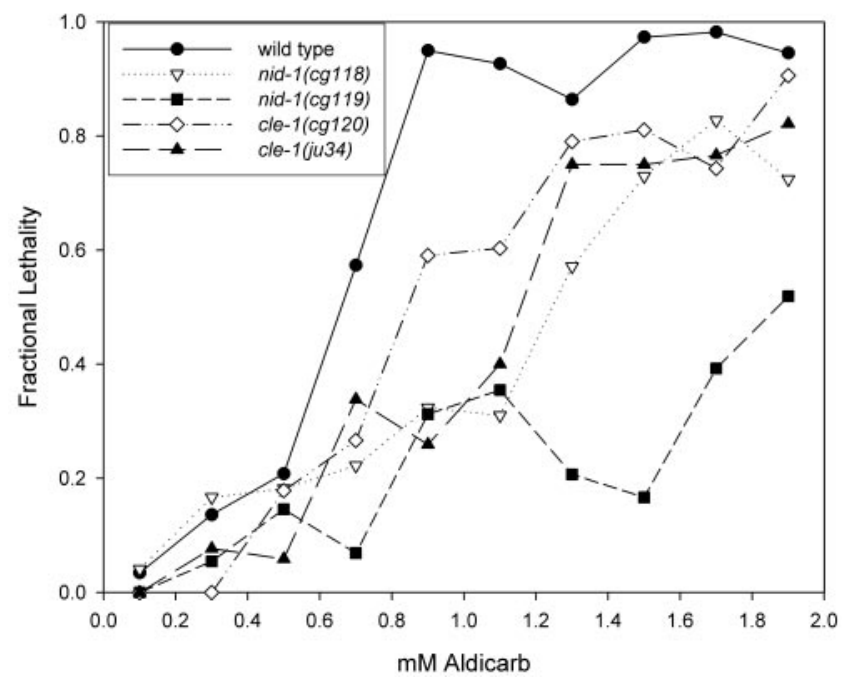

Figure 8. Pharmacological assays of synaptic function. $A$, The average number of eggs laid after $1 \mathrm{hr}$ in the indicated concentrations of the cholinergic agonist levamisole ( $n=20$ for each data point). Both nid-1(cg119) (closed triangles) and nid-1(cg118) (open triangles) animals show reduced response to levamisole at all concentrations tested, with c 1119 exhibiting the greatest resistance. cle-1 animals do not exhibit a decreased average number of eggs laid relative to wild type; however, the range of values for cle- 1 mutants is much greater than for wild type (Table 2). B, Percentage of animals killed by various concentrations of the cholinesterase inhibitor aldicarb. Lethality was scored as an absence of visible pumping and body wall muscle activity. Greater than $90 \%$ of wild-type animals are dead beginning at $0.9 \mathrm{~mm}$ aldicarb. cle- 1 animals show reduced lethality at all concentrations and exhibit only $80-90 \%$ lethality at the highest concentration (1.9 mM). nid-1 (cg119) null mutants exhibit the greatest resistance, with only $50 \%$ lethality at $1.9 \mathrm{~mm}$ aldicarb (Table 3 ).

to aldicarb (Miller et al., 1996). nid-1(cg119) null mutants were highly resistant to aldicarb, with only $50 \%$ of null animals exhibiting lethality at the highest dose tested (Fig. 8, Table 3). Both cle-1 mutants and the nid-1(cg118) animals were also resistant to aldicarb-induced lethality, but to a lesser degree than the nid-1 null mutant. The accumulation of acetylcholine causes paralysis of body wall muscles. All wild-type animals become completely paralyzed at $0.9 \mathrm{~mm}$ aldicarb, whereas nid-1(cg118) and cle-1 animals require $1.7 \mathrm{~mm}$ aldicarb for complete paralysis. Even at 1.9
mM aldicarb, 31\% of nid-1(cg119) continue to exhibit body wall muscle contractions. These data further support the conclusion that synaptic transmission is impaired in these mutant animals.

\section{Discussion}

The nerve ring and nerve cords of C. elegans are closely associated with basement membranes (White et al., 1986). Five major BM molecules have been characterized in C. elegans. Three of these, type IV collagen, perlecan and SPARC, are not found in association with the nerve ring or cords and are not concentrated at muscle edges adjacent to the cords (Graham et al., 1997; Fitzgerald and Schwarzbauer, 1998; Mullen et al., 1999). However, both NID-1 and CLE-1 are found in association with the nervous system in these locations (Kang and Kramer, 2000; Ackley et al., 2001), indicating a potential role for them in nervous system function. Neither NID-1 nor CLE-1 is restricted to the nervous system, because both are detectable at varying levels in most or all basement membranes throughout the animal. Along the nerve cords, CLE-1 and NID-1 show differences in their localizations. Relative to the position of UNC-17, a component of cholinergic presynaptic zones, CLE-1 appears most highly concentrated along the pseudocoelomic face of the nerve cords, i.e., on the dorsal face of the ventral cord and the ventral face of the dorsal cord. In contrast, NID-1 appears most highly concentrated laterally, lying between the tightly apposed nerve cords and body wall muscles.

Although CLE-1 and NID-1 can be found in close association with synaptic markers, their distribution patterns do not directly coincide with synapses. Despite this fact, we have presented evidence that they can affect synapse organization and function. Molecules that can affect synapses need not be synapse specific or localized directly at active zones. For example, the recently described periactive zone (Sone et al., 2000) is separate from the synaptic active zone and does not contain synaptic vesicles. Some widely expressed molecules, e.g., $\beta P S$ integrin and disks large, have been shown to accumulate in the periactive zone and to be required for proper synaptic morphology and function in Drosophila (Beumer et al., 1999; Sone et al., 2000). The presence of CLE-1 and NID-1 in close proximity to synapse-rich regions of the nervous system is consistent with their having a role in synaptic organization.

Functional significance of CLE- 1 and NID-1 association with the nerve cords was supported by the observation that mutations in $c l e-1$ and nid-1 result in distinct defects in synaptic structures. These genes have multiple roles in neurogenesis, including cell migrations and axon guidance (Kim and Wadsworth, 2000; Ackley et al., 2001) and, as shown here, synapse organization. The synaptic organization defects of cle-1 and nid-1 mutants could arise as a secondary consequence of axon guidance defects. However, the cell migration and axon guidance defects of cle-1 and nid-1 mutants have relatively low penetrance, whereas the synaptic defects reported here are fully penetrant. Also, mutants in the two genes display structurally distinct synaptic defects, indicating that axon mispositioning alone cannot account for the synaptic abnormalities. Together these results indicate that interactions with CLE-1 and NID-1 are required at multiple stages of neurogenesis, from the initial events of cell migration through the wiring stage of synaptogenesis. Expression of nid-1 and cle-1 is detectable before the onset of the morphogenetic phase of embryogenesis, before the formation of the nervous system (Kang and Kramer, 2000; Ackley et al., 2001).

We observe defects in both presynaptic and postsynaptic structures. Previous work has shown that molecules that function 
Table 2. Egg laying in response to levamisole

\begin{tabular}{|c|c|c|c|c|c|c|}
\hline & \multicolumn{6}{|c|}{ Levamisole concentration } \\
\hline & $0 \mu \mathrm{m}^{A}$ & $3 \mu \mathrm{M}$ & $6 \mu \mathrm{m}$ & $12 \mu \mathrm{m}$ & $25 \mu \mathrm{M}$ & $50 \mu \mathrm{M}$ \\
\hline Wild type & $0.4 \pm 0.8$ & $2.8 \pm 2.0$ & $5.0 \pm 2.4$ & $6.3 \pm 1.6$ & $8.6 \pm 2.4$ & $13.7 \pm 1.8$ \\
\hline Range & $0-3$ & $0-8$ & $2-9$ & $3-8$ & $5-13$ & $10-16$ \\
\hline nid-1(cg118) & $0.1 \pm 0.2$ & $0.4 \pm 0.8$ & $0.8 \pm 1.7$ & $3.9 \pm 5.6$ & $4.2 \pm 3.4$ & $7.7 \pm 4.6$ \\
\hline Range & $0-1$ & $0-2$ & $0-5$ & $0-18$ & $0-11$ & $0-16$ \\
\hline nid-1(cg119) & $0.3 \pm 0.9$ & $0.4 \pm 0.9$ & $2.3 \pm 3.0$ & $2.2 \pm 2.1$ & $2.9 \pm 3.9$ & $6.0 \pm 3.5$ \\
\hline Range & $0-3$ & $0-3$ & $0-10$ & $0-6$ & $0-12$ & $0-15$ \\
\hline cle-1(cg120) & $0.3 \pm 0.7$ & $4.7 \pm 4.0$ & $7.8 \pm 4.3$ & $9.6 \pm 4.9$ & $13.1 \pm 7.1$ & $10.3 \pm 5.7$ \\
\hline Range & $0-3$ & $0-12$ & $0-16$ & $0-20$ & $1-24$ & $0-21$ \\
\hline cle-1(ju34) & $0.0 \pm 0.0$ & $1.0 \pm 2.9$ & $4.1 \pm 4.3$ & $8.6 \pm 4.8$ & $9.2 \pm 5.6$ & $10.1 \pm 3.3$ \\
\hline Range & $0-0$ & $0-12$ & $0-13$ & $0-18$ & $0-19$ & $3-17$ \\
\hline
\end{tabular}

${ }^{a}$ Average number of eggs laid $\pm S D$ after $1 \mathrm{hr}$ exposure to indicated concentrations of levamisole. The range of values is also indicated $(n=20)$.

Table 3. Aldicarb-induced lethality

\begin{tabular}{|c|c|c|c|c|c|c|c|c|c|c|}
\hline & Aldicarb & tration & & & & & & & & \\
\hline & $0.1 \mathrm{~mm}$ & & $0.3 \mathrm{~mm}$ & & $0.5 \mathrm{~mm}$ & & $0.7 \mathrm{~mm}$ & & $0.9 \mathrm{~mm}$ & \\
\hline & $\operatorname{Dead}^{a}$ & Moving $^{b}$ & Dead & Moving & Dead & Moving & Dead & Moving & Dead & Moving \\
\hline Wild type & 3 & 93 & 14 & 77 & 21 & 67 & 57 & 31 & 95 & 0 \\
\hline nid-1(cg118) & 4 & 96 & 17 & 61 & 18 & 48 & 22 & 22 & 32 & 15 \\
\hline nid-1(cg119) & 0 & 100 & 6 & 78 & 15 & 63 & 7 & 76 & 31 & 44 \\
\hline cle-1(cg120) & 0 & 100 & 0 & 70 & 18 & 64 & 27 & 70 & 59 & 24 \\
\hline cle-1(ju34) & 0 & 100 & 8 & 92 & 6 & 88 & 34 & 34 & 26 & 41 \\
\hline & $1.1 \mathrm{~mm}$ & & $1.3 \mathrm{~mm}$ & & $1.5 \mathrm{~mm}$ & & $1.7 \mathrm{~mm}$ & & $1.9 \mathrm{~mm}$ & \\
\hline Wild type & 93 & 0 & 86 & 2 & 97 & 0 & 98 & 2 & 95 & 0 \\
\hline nid-1(cg118) & 31 & 17 & 57 & 7 & 73 & 3 & 83 & 3 & 72 & 0 \\
\hline nid-1(cg119) & 36 & 39 & 21 & 38 & 17 & 47 & 39 & 21 & 52 & 31 \\
\hline cle-1(cg120) & 60 & 14 & 79 & 7 & 81 & 11 & 74 & 8 & 91 & 6 \\
\hline cle-1(ju34) & 40 & 20 & 75 & 8 & 75 & 8 & 77 & 10 & 82 & 0 \\
\hline
\end{tabular}

a Percentage of dead animals after $8 \mathrm{hr}$ in indicated concentrations of aldicarb $(n=25-66)$.

${ }^{b}$ Percentage of animals exhibiting pharyngeal pumping and body wall muscle activity after $8 \mathrm{hr}$ in indicated concentrations of aldicarb. Animals not scored as Dead or Moving exhibited only pharyngeal pumping.

specifically in the presynaptic neurons can affect the organization of the postsynaptic structure (Zhen and Jin, 1999), although mechanistically it was unclear how this occurred. Our data suggest that the coordinate formation could be mediated, at least in part, by components of the ECM. In mice, $\alpha 4$ chain-containing laminins are concentrated between the presynaptic active zone and the postsynaptic receptor clefts, which are not properly aligned in mice lacking the laminin $\alpha 4$ chain (Patton et al., 2001).

The uncoordinated movement seen in thrashing assays and the altered responses to levamisole, a cholinergic agonist, and aldicarb, a cholinesterase inhibitor, suggest that synaptic transmission is impaired in cle-1 and nid-1 mutant animals. Because these mutants have axon-positioning and synapse-organization abnormalities, the defects observed in these assays could result from either or both of these abnormalities. Genetic screens in $C$. elegans for resistance to cholinesterase inhibitors have been very successful at identifying molecules that regulate synaptic transmission but have not reportedly identified molecules that affect axon guidance (Jorgensen et al., 1995; Miller et al., 1996). Neither cle-1 nor nid-1 mutants are as strongly resistant as the strongest mutations identified in these screens. However, their altered responses to cholinergic modulators most likely reflect defects in synaptic transmission.

The distinct structural defects in synaptic structures seen in cle-1 and nid-1 mutants suggest that these molecules have specific roles in synapse organization. In cle-1 mutants, synaptic structures in the dorsal and ventral cords are enlarged and spaced farther apart than in wild-type animals, with an apparent reduc- tion in number of synapses. Both cle-1 mutations, $\operatorname{cg} 120$ and ju34, result in loss of the type XVIII collagen NC1 domain, suggesting that the loss of this domain may cause the defects observed. The $\mathrm{NC1}$ /endostatin domain has been shown to bind integrins (Rehn et al., 2001) and could interact directly with cells during organization of synapses. The $\mathrm{NC1}$ domain has been shown to stimulate cell motility (Kuo et al., 2001) and bind the laminin-nidogen complex, as well as other ECM molecules (Sasaki et al., 1998). Thus, the defects of synaptic organization seen in cle-1 mutants could be caused by problems in the migrations of presynaptic or postsynaptic cells or in the presentation of the ECM to these cells during synaptogenesis.

The nid-1 null mutant shows slightly different defects in the dorsal and ventral cords. In both, synapses are elongated along the length of the cord and more closely spaced than normal. Along the ventral cord the puncta appear narrowed and fragmented, whereas on the dorsal cord an increase in size is observed. The reason for this difference is unknown. One possibility is that the VD motor neurons, which form the ventral synapses, and the DD motor neurons, which form the dorsal synapses, respond differently to the absence of nidogen. The cg118 NID$1(\Delta G 2)$ mutants show more similar defects in the dorsal and ventral cords, with less severely enlarged and aberrantly spaced puncta than seen in the null mutants.

Nidogen has been suggested to link together laminin and collagen IV or perlecan polymer networks in basement membranes on the basis of the binding of its G2 domain to type IV collagen and perlecan and of its G3 domain to laminin (Aumailley et al., 
1993; Reinhardt et al., 1993). We generally observed less severe defects in NID- $1(\Delta G 2)$ animals relative to the null animals, particularly in resistance to levamisole and aldicarb. NID-1( $\Delta$ G2) was also previously shown to result in less severe loss of fecundity than the NID-1 null (Kang and Kramer, 2000). Together these results indicate that loss of the G2 domain causes only a partial reduction in nidogen function and argues that the proposed linking role for nidogen cannot account for all of its functions. The finding of neurological defects in nidogen-1/entactin-1-deficient mice (Dong et al., 2002) suggests that the axonal and synaptic defects described for C. elegans nid-1 mutants may also occur in these mice.

Synapse formation is a highly complex process that requires multiple dynamic interactions between neurons and target cells. We have shown that CLE- 1 and NID-1 are associated with the nervous system in C. elegans and are required for proper synapse organization and function. Understanding how these molecules function to specify synaptic formation is an ongoing challenge. This is the first demonstration of a role for these proteins during synaptogenesis, but reinforces the utility of $C$. elegans for identifying novel players in the process of synaptogenesis.

\section{References}

Ackley BD, Crew JR, Elamaa H, Pihlajaniemi T, Kuo CJ, Kramer JM (2001) The NC1/endostatin domain of Caenorhabditis elegans type XVIII collagen affects cell migration and axon guidance. J Cell Biol 152:1219-1232.

Alfonso A, Grundahl K, Duerr JS, Han HP, Rand JB (1993) The Caenorhabditis elegans unc-17 gene: a putative vesicular acetylcholine transporter. Science 261:617-619.

Allamand V, Sunada Y, Salih MA, Straub V, Ozo CO, Al-Turaiki MH, Akbar M, Kolo T, Colognato H, Zhang X, Sorokin LM, Yurchenco PD, Tryggvason K, Campbell KP (1997) Mild congenital muscular dystrophy in two patients with an internally deleted laminin alpha2-chain. Hum Mol Genet 6:747-752.

Aumailley M, Battaglia C, Mayer U, Reinhardt D, Nischt R, Timpl R, Fox JW (1993) Nidogen mediates the formation of ternary complexes of basement membrane components. Kidney Int 43:7-12.

Bamber BA, Beg AA, Twyman RE, Jorgensen EM (1999) The Caenorhabditis elegans unc-49 locus encodes multiple subunits of a heteromultimeric GABA receptor. J Neurosci 19:5348-5359.

Bettinger JC, Lee K, Rougvie AE (1996) Stage-specific accumulation of the terminal differentiation factor LIN-29 during Caenorhabditis elegans development. Development 122:2517-2527.

Beumer KJ, Rohrbough J, Prokop A, Broadie K (1999) A role for PS integrins in morphological growth and synaptic function at the postembryonic neuromuscular junction of Drosophila. Development 126:5833-5846.

Brenner S (1974) The genetics of Caenorhabditis elegans. Genetics 77:71-94.

Burkin DJ, Kaufman SJ (1999) The alpha7betal integrin in muscle development and disease. Cell Tissue Res 296:183-190.

Burkin DJ, Wallace GQ, Nicol KJ, Kaufman DJ, Kaufman SJ (2001) Enhanced expression of the alpha 7 beta 1 integrin reduces muscular dystrophy and restores viability in dystrophic mice. J Cell Biol 152:1207-1218.

Campanelli JT, Ferns M, Hoch W, Rupp F, von Zastrow M, Hall Z, Scheller RH (1992) Agrin: a synaptic basal lamina protein that regulates development of the neuromuscular junction. Cold Spring Harbor Symp Quant Biol 57:461-472.

Chiu AY, Ko J (1994) A novel epitope of entactin is present at the mammalian neuromuscular junction. J Neurosci 14:2809-2817.

Cohn RD, Campbell KP (2000) Molecular basis of muscular dystrophies. Muscle Nerve 23:1456-1471.

Colognato H, Yurchenco PD (1999) The laminin alpha2 expressed by dystrophic dy $(2 \mathrm{~J})$ mice is defective in its ability to form polymers. Curr Biol 9:1327-1330.

Colognato H, Yurchenco PD (2000) Form and function: the laminin family of heterotrimers. Dev Dyn 218:213-234.

Crump JG, Zhen M, Jin Y, Bargmann CI (2001) The SAD-1 kinase regulates presynaptic vesicle clustering and axon termination. Neuron 29:115-129.

Dong L, Chen Y, Lewis M, Hsieh JC, Reing J, Chaillet JR, Howell CY, Melhem M, Inoue S, Kuszak JR, DeGeest K, Chung AE (2002) Neurologic defects and selective disruption of basement membranes in mice lacking entactin-1/nidogen-1. Lab Invest 82:1617-1630.

Durkin ME, Chakravarti S, Bartos BB, Liu SH, Friedman RL, Chung AE (1988) Amino acid sequence and domain structure of entactin. Homology with epidermal growth factor precursor and low density lipoprotein receptor. J Cell Biol 107:2749-2756.

Eklund L, Piuhola J, Komulainen J, Sormunen R, Ongvarrasopone C, Fassler R, Muona A, Ilves M, Ruskoaho H, Takala TE, Pihlajaniemi T (2001) Lack of type XV collagen causes a skeletal myopathy and cardiovascular defects in mice. Proc Natl Acad Sci USA 98:1194-1199.

Finney M, Ruvkun G (1990) The unc-86 gene product couples cell lineage and cell identity in C. elegans. Cell 63:895-905.

Fitzgerald MC, Schwarzbauer JE (1998) Importance of the basement membrane protein SPARC for viability and fertility in Caenorhabditis elegans. Curr Biol 8:1285-1288.

Fukai N, Eklund L, Marneros AG, Oh SP, Keene DR, Tamarkin L, Niemela M, Ilves M, Li E, Pihlajaniemi T, Olsen BR (2002) Lack of collagen XVIII/ endostatin results in eye abnormalities. EMBO J 21:1535-1544.

Graham PL, Johnson JJ, Wang S, Sibley MH, Gupta MC, Kramer JM (1997) Type IV collagen is detectable in most, but not all, basement membranes of Caenorhabditis elegans and assembles on tissues that do not express it. J Cell Biol 137:1171-1183.

Gu T, Orita S, Han M (1998) Caenorhabditis elegans SUR-5, a novel but conserved protein, negatively regulates LET-60 Ras activity during vulval induction. Mol Cell Biol 18:4556-4564.

Guo XD, Johnson JJ, Kramer JM (1991) Embryonic lethality caused by mutations in basement membrane collagen of C. elegans. Nature 349:707-709.

Gupta MC, Graham PL, Kramer JM (1997) Characterization of alpha 1(IV) collagen mutations in Caenorhabditis elegans and the effects of alpha 1 and alpha 2(IV) mutations on type IV collagen distribution. J Cell Biol 137:1185-1196.

Hallam SJ, Jin Y (1998) lin-14 regulates the timing of synaptic remodelling in Caenorhabditis elegans. Nature 395:78-82.

Huang X, Cheng H-J, Tessier-Lavigne M, Jin Y (2002) MAX-1, a novel PH/ Myth4/FERM domain cytoplasmic protein implicated in netrinmediated axon repulsion. Neuron 34:563-576.

Jin Y, Jorgensen E, Hartwieg E, Horvitz HR (1999) The Caenorhabditis elegans gene unc-25 encodes glutamic acid decarboxylase and is required for synaptic transmission but not synaptic development. J Neurosci 19:539-548.

Jorgensen EM, Hartwieg E, Schuske K, Nonet ML, Jin YS, Horvitz HR (1995) Defective recycling of synaptic vesicles in synaptotagmin mutants of Caenorhabditis elegans. Nature 378:196-199.

Kang SH, Kramer JM (2000) Nidogen is nonessential and not required for normal type IV collagen localization in Caenorhabditis elegans. Mol Biol Cell 11:3911-3923.

Kim J, Poole DS, Waggoner LE, Kempf A, Ramirez DS, Treschow PA, Schafer WR (2001) Genes affecting the activity of nicotinic receptors involved in Caenorhabditis elegans egg-laying behavior. Genetics 157:1599-1610.

Kim S, Wadsworth WG (2000) Positioning of longitudinal nerves in C. elegans by nidogen. Science 288:150-154.

Kivirikko S, Heinamaki P, Rehn M, Honkanen N, Myers JC, Pihlajaniemi T (1994) Primary structure of the alpha 1 chain of human type XV collagen and exon-intron organization in the $3^{\prime}$ region of the corresponding gene. J Biol Chem 269:4773-4779.

Kohfeldt E, Sasaki T, Gohring W, Timpl R (1998) Nidogen-2: a new basement membrane protein with diverse binding properties. J Mol Biol 282:99-109.

Koushika SP, Richmond JE, Hadwiger G, Weimer RM, Jorgensen EM, Nonet ML (2001) A post-docking role for active zone protein Rim. Nat Neurosci 4:997-1005.

Kuhn K (1995) Basement membrane (type IV) collagen. Matrix Biol 14:439-445.

Kuo CJ, LaMontagne KR Jr, Garcia-Cardena G, Ackley BD, Kalman D, Park S, Christofferson R, Kamihara J, Ding YH, Lo KM, Gillies S, Folkman J, Mulligan RC, Javaherian K (2001) Oligomerization-dependent regulation of motility and morphogenesis by the collagen XVIII NC1/endostatin domain. J Cell Biol 152:1233-1246.

Lewis JA, Wu CH, Berg H, Levine JH (1980a) The genetics of levamisole resistance in the nematode Caenorhabditis elegans. Genetics 95:905-928.

Lewis JA, Wu CH, Levine JH, Berg H (1980b) Levamisole-resistant mutants of the nematode Caenorhabditis elegans appear to lack pharmacological acetylcholine receptors. Neuroscience 5:967-989.

Lewis JA, Elmer JS, Skimming J, McLafferty S, Fleming J, McGee T (1987) 
Cholinergic receptor mutants of the nematode Caenorhabditis elegans. J Neurosci 7:3059-3071.

Mackenzie JM Jr, Garcea RL, Zengel JM, Epstein HF (1978) Muscle development in Caenorhabditis elegans: mutants exhibiting retarded sarcomere construction. Cell 15:751-762.

Mann K, Deutzmann R, Aumailley M, Timpl R, Raimondi L, Yamada Y, Pan TC, Conway D, Chu ML (1989) Amino acid sequence of mouse nidogen, a multidomain basement membrane protein with binding activity for laminin, collagen IV and cells. EMBO J 8:65-72.

Miller KG, Alfonso A, Nguyen M, Crowell JA, Johnson CD, Rand JB (1996) A genetic selection for Caenorhabditis elegans synaptic transmission mutants. Proc Natl Acad Sci USA 93:12593-12598.

Mullen GP, Rogalski TM, Bush JA, Gorji PR, Moerman DG (1999) Complex patterns of alternative splicing mediate the spatial and temporal distribution of perlecan/UNC-52 in Caenorhabditis elegans. Mol Biol Cell 10:3205-3221.

Murshed M, Smyth N, Miosge N, Karolat J, Krieg T, Paulsson M, Nischt R (2000) The absence of nidogen 1 does not affect murine basement membrane formation. Mol Cell Biol 20:7007-7012.

Musso O, Rehn M, Saarela J, Theret N, Lietard J, Hintikka, Lotrian D, Campion JP, Pihlajaniemi T, Clement B (1998) Collagen XVIII is localized in sinusoids and basement membrane zones and expressed by hepatocytes and activated stellate cells in fibrotic human liver. Hepatology 28:98-107.

Myers JC, Dion AS, Abraham V, Amenta PS (1996) Type XV collagen exhibits a widespread distribution in human tissues but a distinct localization in basement membrane zones. Cell Tissue Res 286:493-505.

Nguyen QT, Son YJ, Sanes JR, Lichtman JW (2000) Nerve terminals form but fail to mature when postsynaptic differentiation is blocked: in vivo analysis using mammalian nerve-muscle chimeras. J Neurosci 20:6077-6086.

Noakes PG, Gautam M, Mudd J, Sanes JR, Merlie JP (1995) Aberrant differentiation of neuromuscular junctions in mice lacking s-laminin/laminin beta 2. Nature 374:258-262.

Nonaka I (1998) Animal models of muscular dystrophies. Lab Anim Sci 48:8-17.

Nonet ML (1999) Visualization of synaptic specializations in live C. elegans with synaptic vesicle protein-GFP fusions. J Neurosci Methods 89:33-40.

Nonet ML, Grundahl K, Meyer BJ, Rand JB (1993) Synaptic function is impaired but not eliminated in C. elegans mutants lacking synaptotagmin. Cell 73:1291-1305.

Patton BL, Chiu AY, Sanes JR (1998) Synaptic laminin prevents glial entry into the synaptic cleft. Nature 393:698-701.

Patton BL, Cunningham JM, Thyboll J, Kortesmaa J, Westerblad H, Edstrom L, Tryggvason K, Sanes JR (2001) Properly formed but improperly localized synaptic specializations in the absence of laminin alpha4. Nat Neurosci 4:597-604.

Peng HB, Xie H, Rossi SG, Rotundo RL (1999) Acetylcholinesterase clustering at the neuromuscular junction involves perlecan and dystroglycan. J Cell Biol 145:911-921.

Rehn M, Pihlajaniemi T (1994) Alpha 1(XVIII), a collagen chain with frequent interruptions in the collagenous sequence, a distinct tissue distribution, and homology with type XV collagen. Proc Natl Acad Sci USA 91:4234-4238.

Rehn M, Veikkola T, Kukk-Valdre E, Nakamura H, Ilmonen M, Lombardo C, Pihlajaniemi T, Alitalo K, Vuori K (2001) Interaction of endostatin with integrins implicated in angiogenesis. Proc Natl Acad Sci USA 98:1024-1029.
Reinhardt D, Mann K, Nischt R, Fox JW, Chu ML, Krieg T, Timpl R (1993) Mapping of nidogen binding sites for collagen type IV, heparan sulfate proteoglycan, and zinc. J Biol Chem 268:10881-10887.

Rogalski TM, Gilchrist EJ, Mullen GP, Moerman DG (1995) Mutations in the unc-52 gene responsible for body wall muscle defects in adult Caenorhabditis elegans are located in alternatively spliced exons. Genetics 139:159-169.

Sanes JR (1997) Genetic analysis of postsynaptic differentiation at the vertebrate neuromuscular junction. Curr Opin Neurobiol 7:93-100.

Sanes JR, Lichtman JW (1999) Development of the vertebrate neuromuscular junction. Annu Rev Neurosci 22:389-442.

Sanes JR, Yamagata M (1999) Formation of lamina-specific synaptic connections. Curr Opin Neurobiol 9:79-87.

Sasaki T, Fukai N, Mann K, Gohring W, Olsen BR, Timpl R (1998) Structure, function and tissue forms of the C-terminal globular domain of collagen XVIII containing the angiogenesis inhibitor endostatin. EMBO J 17:4249-4256.

Schaefer AM, Hadwiger GD, Nonet ML (2000) rpm-1, a conserved neuronal gene that regulates targeting and synaptogenesis in C. elegans. Neuron 26:345-356.

Schymeinsky J, Nedbal S, Miosge N, Poschl E, Rao C, Beier DR, Skarnes WC, Timpl R, Bader BL (2002) Gene structure and functional analysis of the mouse nidogen-2 gene: nidogen-2 is not essential for basement membrane formation in mice. Mol Cell Biol 22:6820-6830.

Sertie AL, Sossi V, Camargo AA, Zatz M, Brahe C, Passos-Bueno MR (2000) Collagen XVIII, containing an endogenous inhibitor of angiogenesis and tumor growth, plays a critical role in the maintenance of retinal structure and in neural tube closure (Knobloch syndrome). Hum Mol Genet 9:2051-2058.

Sibley MH, Graham PL, von Mende N, Kramer JM (1994) Mutations in the $\alpha 2$ (IV) basement membrane collagen gene of Caenorhabditis elegans produce phenotypes of differing severities. EMBO J 13:3278-3285.

Sone M, Suzuki E, Hoshino M, Hou D, Kuromi H, Fukata M, Kuroda S, Kaibuchi K, Nabeshima Y, Hama C (2000) Synaptic development is controlled in the periactive zones of Drosophila synapses. Development 127:4157-4168.

Sunada Y, Bernier SM, Utani A, Yamada Y, Campbell KP (1995) Identification of a novel mutant transcript of laminin alpha 2 chain gene responsible for muscular dystrophy and dysmyelination in dy2J mice. Hum Mol Genet 4:1055-1061.

Timpl R (1994) Proteoglycans of basement membranes. EXS 70:123-144.

Waggoner LE, Dickinson KA, Poole DS, Tabuse Y, Miwa J, Schafer WR (2000) Long-term nicotine adaptation in Caenorhabditis elegans involves PKC-dependent changes in nicotinic receptor abundance. J Neurosci 20:8802-8811.

White JG, Southgate E, Thomson JN, Brenner S (1986) The structure of the nervous system of Caenorhabditis elegans. Philos Trans R Soc Lond B Biol Sci 314:1-340.

Yurchenco PD, Schittny JC (1990) Molecular architecture of basement membranes. FASEB J 4:1577-1590.

Zhen M, Jin Y (1999) The liprin protein SYD-2 regulates the differentiation of presynaptic termini in C. elegans. Nature 401:371-375.

Zhen M, Huang X, Bamber B, Jin Y (2000) Regulation of presynaptic terminal organization by C. elegans RPM-1, a putative guanine nucleotide exchanger with a RING-H2 finger domain. Neuron 26:331-343. 